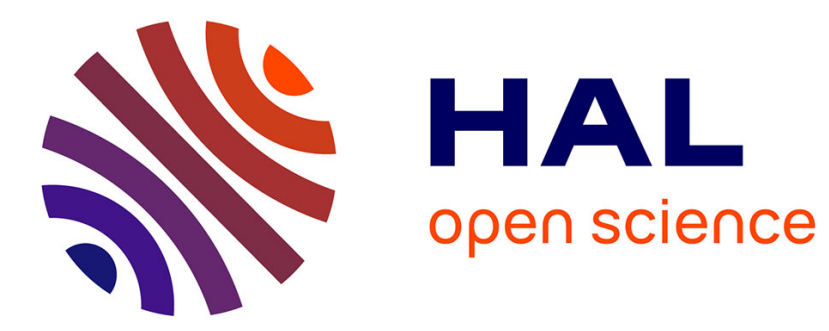

\title{
Plastic deformation and residual stresses in amorphous silica pillars under uniaxial loading
}

\author{
R. Lacroix, G. Kermouche, J. Teisseire, E. Barthel
}

\section{To cite this version:}

R. Lacroix, G. Kermouche, J. Teisseire, E. Barthel. Plastic deformation and residual stresses in amorphous silica pillars under uniaxial loading. Acta Materialia, 2012, 60 (15), pp.5555-5566. 10.1016/j.actamat.2012.07.016 . hal-00746956

\section{HAL Id: hal-00746956 \\ https://hal.science/hal-00746956}

Submitted on 4 Oct 2021

HAL is a multi-disciplinary open access archive for the deposit and dissemination of scientific research documents, whether they are published or not. The documents may come from teaching and research institutions in France or abroad, or from public or private research centers.
L'archive ouverte pluridisciplinaire HAL, est destinée au dépôt et à la diffusion de documents scientifiques de niveau recherche, publiés ou non, émanant des établissements d'enseignement et de recherche français ou étrangers, des laboratoires publics ou privés. 


\title{
Plastic deformation and residual stresses in amorphous silica pillars under uniaxial loading
}

\author{
R. Lacroix ${ }^{\mathrm{a}, \mathrm{b}, *}$, G. Kermouche ${ }^{\mathrm{b}}$, J. Teisseire $^{\mathrm{c}}$, E. Barthel ${ }^{\mathrm{c}}$ \\ ${ }^{a}$ Université de Lyon, ENISE, LTDS, UMR 5513, 42023 Saint-Etienne Cedex 2, France \\ ${ }^{\mathrm{b}}$ École Nationale Supérieure des Mines de Saint-Étienne, SMS, UMR CNRS 5146, 158, cours Fauriel 42023 Saint-Etienne Cedex 2, France \\ ${ }^{\mathrm{c}}$ Surface du Verre et Interfaces, CNRS/Saint-Gobain, UMR 125, 93303 Aubervilliers Cedex, France
}

\begin{abstract}
We have carried out uniaxial compression of micron-scale amorphous silica pillars. We have measured load-displacement curves and observed the morphology of the pillars after unloading, providing strong evidence for large plastic deformations. Minor cracking is also observed, with a well-defined pattern. We find that the van Mises stress in compression is comparable to the intrinsic tensile strength of silica. Precise analysis of the deformation of the pillars has been carried out by finite element modeling (FEM) using the constitutive equation determined previously (G. Kermouche et al., Acta Materialia, 56 (2008) 3222), which quantitatively takes into account densification, shear flow and strain hardening. The residual stress distribution we predict by FEM matches the observed crack pattern well. Finally the calculated stress fields in pillar compression and cone indentation are compared. We propose an interpretation of the contrasts in terms of confinement.
\end{abstract}

Keywords: Amorphous silica; Micropillars; Yield surface; Nanoindentation; Finite element analysis

\section{Introduction}

Silicate glasses are brittle - a claim substantiated by our daily experience. However, it has also been known for years that they are ductile at small scales [1]. For example, Marsh pointed out the close similarity between rows of indents in steel and in plate glass as early as 1964 [2]. We believe that progress in our understanding of this ductility of silicate glasses is one of the main avenues towards the reduction of their fragility.

There may be two distinct relations between fragility and ductility in silicate glasses. On the one hand, practical strength is limited by surface flaws [3]. Many of these flaws are generated by contact loading of the surface, in which

\footnotetext{
* Corresponding author at: Université de Lyon, ENISE, LTDS, UMR 5513, 42023 Saint-Etienne Cedex 2, France.

E-mail address: remi.lacroix@saint-gobain.com (R. Lacroix).
}

plastic deformation is involved at smaller length scales. Understanding the ductility of silicates may help curb the formation of strength-threatening flaws. On the other hand, there is the question of the intrinsic tensile strength of silicates. Many accurate measurements of the tensile strength of silicate glasses have been obtained over the last 20 years. It is significantly dependent upon composition and environment, but generally speaking the tensile strength reaches very large values. For silica, accepted values at room temperature are 5.5-6 GPa under an ambient atmosphere and 10-12 GPa under inert conditions [4,5]. At such levels of stress the question of the rupture mechanism arises, both for initiation and propagation: is it a purely brittle rupture (bond by bond) or does collective reorganization (i.e. ductility) play a role? Fundamental comprehension of the ductility in silicate glasses would help to answer this question, which is relevant for possible relations between plastic yield stress and intrinsic tensile strength and also between fracture energy and surface 
energy. For this reason, a sound understanding of the plasticity of silicates is needed.

The most fundamental question is: what are the exact irreversible deformation mechanisms that can give rise to plasticity in an amorphous material [6]. In the absence of periodicity, dislocations are unavailable and shear flow must rely on other processes, such as more local plastic rearrangements $[7,8]$. Moreover, the continuous distribution of interatomic distances allows for specific irreversible evolutions of the structure during permanent deformation. In the case of silica, there is a $20 \%$ free volume that can be suppressed by these local rearrangements: a large permanent volumetric strain (or densification) can be observed during plastic deformation [9-11].

The second question is methodological: experimentally, how can we investigate the plastic deformation of silicates since the typical length scales involved are so small? As a rule of thumb, the brittle to ductile transition occurs around the micron scale for amorphous silicates. This is why, although early experiments successfully identified the phenomenology of the irreversible deformation processes such as densification [9] and shear flow [2,12] during sharp indentation, they could not quantify the constitutive response of the material. In this area, significant progress was made once well-defined stress-strain experiments at the micron scale became possible. This progress was due to the development of microfabrication tools and local strain measurement capabilities. First, data for accurate identification of the yield surface were obtained by diamond anvil cell (DAC) measurements, under a loading close to pure hydrostatic pressure [13]. The relation between maximum hydrostatic pressure $p$ and final density has been measured up to $20 \mathrm{GPa}$ for amorphous silica $[14,15]$ and soda-lime glass $[16,17]$. Since hardening occurs as densification increases $[16,14]$, shear flow dominates the deformation process once full densification is achieved [18]. The coupling between densification and the equivalent von Mises stress was investigated in detail through indentation experiments. Nanoindentation experiments have been analyzed by Xin and Lambropoulos [19] using finite element modeling (FEM). Mappings of the density distribution [14] in Vickers indents by Raman spectroscopy were shown to provide significantly richer data for the identification of the yield surface. Based on these data, a constitutive equation has been proposed [20] which includes the effects of shear flow, densification and strain hardening, and accounts simultaneously for the DAC, the nanoindentation load-displacement curve and the density maps.

In indentation, complex stress states come as a distribution, which is not easy to unravel. In order to interrogate the yield properties accurately, other experiments with purer loadings are needed. Inspiration can be derived from recent developments in the area of the plastic response of metals at the small scale: as an approximation to uniaxial loading, micropillar experiments have been proposed. Under uniaxial loading, the relation $q / p=3$ is obeyed, where $q$ is the equivalent von Mises stress. Micropillar compression has been widely used for the investigation of the response of metallic single crystals and metallic glasses [21-24], and has proved extremely valuable in the characterization of strength and flow behavior. However, attention has been drawn to the unavoidable deviations from the ideal homogeneous uniaxial stress state due to geometrical approximations and imperfect boundary conditions [25-28]. It is now clear that reliable conclusions on intrinsic material response can be derived only when the details of the experimental set-up are taken into account explicitly in the analysis.

For amorphous silica, we have recently shown that silicate glasses are very suitable for micropillar compression because the ratio of the yield stress to Young's modulus is comparatively large [29]. In the present paper, we investigate plastic flow in the compression of amorphous silica micropillars. We demonstrate the experimental conditions under which controlled plastic flow can be obtained with high reproducibility, without failure of the pillar, generating only minor cracking with a well-defined pattern. We demonstrate that, under these loading conditions, the plastic flow takes place at a von Mises stress of about 6.9 GPa. An in-depth analysis of the pillar response by FEM supports our interpretation: deviations from ideal uniaxial conditions are considered in detail and provide explanations for heterogeneous plastic flow in the pillar. Our results suggest that this flow is nearly volume-conservative. We also calculate the residual stress distribution and demonstrate that it is consistent with the observed crack pattern. Finally, the results are compared with cone indentation of a flat silica surface and the contrast with pillar compression is discussed in terms of confinement.

\section{Material and methods}

\subsection{Pillars-material and morphology}

Amorphous silica wafers ( 3 inches, polished on one side, (7.64 mm) thickness, Neyco SA (Paris) France) were etched by reactive ion etching (RIE) [29]. Note that quoting the diameter in inches is standard practice in this field. The resulting micropillars were $4.75 \mu \mathrm{m}$ high and $3.1 \mu \mathrm{m}$ in diameter at the upper section. Under the present etching conditions, a taper angle of about $4^{\circ}$ is observed (Fig. 1).

Individual micropillars were axially loaded with a flat punch $(11 \mu \mathrm{m}$ diameter, Fig. 2) in a nanoindenter (Agilent NanoXP), as described in Ref. [29]. The resolutions are $\pm 50 \mu \mathrm{N}$ for the load and $\pm 0.01 \mathrm{~nm}$ for the displacement. Loading and unloading are carried out at the same rate of $0.1 \mathrm{mN} \mathrm{s}^{-1}$, which generates a strain rate of about $1.7 \times 10^{-3} \mathrm{~s}^{-1}$ during the elastic regime.

Following the conclusions of our previous experiments [29], we have optimized the geometry of the experiment to limit the buckling instability of the pillar. This is why a rather low aspect ratio $H / D=1.5$ is used, increasing the stability of the pillar [25]. In addition, the loading moments are minimized. The sample surface is aligned 

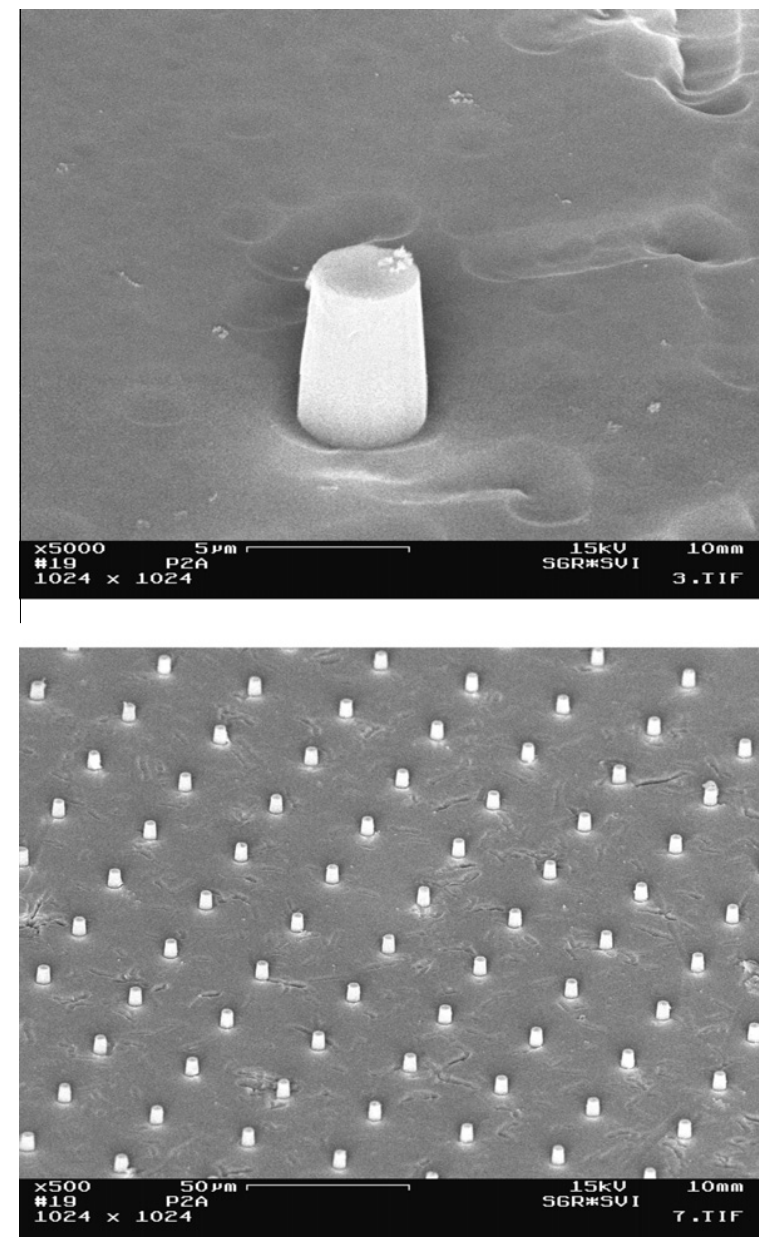

Fig. 1. Field emission gun scanning electron microscope (FEG-SEM) images of typical amorphous silica micropillars. The array was fabricated by RIE.
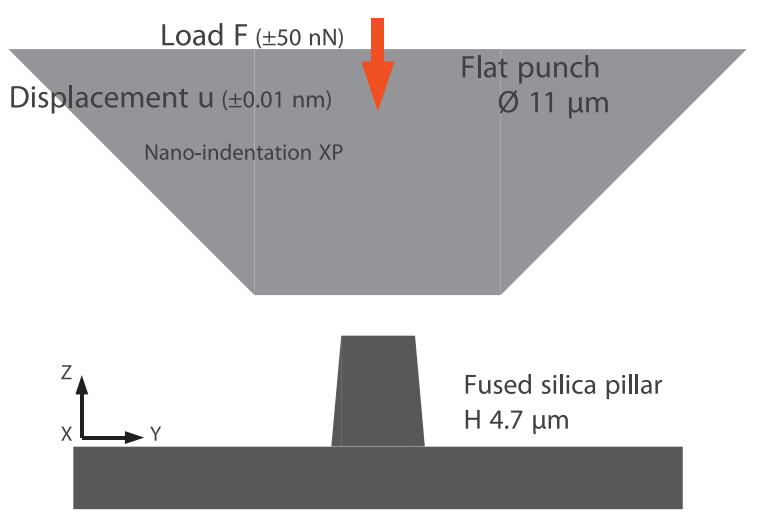

Fig. 2. Geometry of the micropillar compression set-up in the nanoindenter.

precisely with the vertical axis of the indenter using a custom-made tilt stage (Michalex SARL, Orsay, France), as proposed by Uchic et al. [21]. Alignment is performed using the built-in optical microscope (field depth $1 \mu \mathrm{m}$ ) and deemed adequate when the sample surface is in focus on all four sides of the sample (size $20 \mathrm{~mm} \times 20 \mathrm{~mm}$ ). Special care is also given to the calibration of the position in the horizontal plane to ensure that the pillar makes contact with the flat punch at the center.

\subsection{Measurement procedure}

During compression, the displacement effectively applied to the top surface of the pillar $u_{t i p}$ is evaluated as follows. Even with optimized alignment, an accommodation range is observed over a few hundred nanometers (Fig. 3). In order to accurately define the origin of displacement, a $15 \mathrm{mN}$ preload is first applied (Fig. 3, section a). Unloading (section b) and reloading (section c) demonstrates that accommodation has been suppressed and that the origin of displacement $u_{0}$ is now well defined (end of segment b, Fig. 3). In addition, two other corrections are needed. The first correction is for the frame stiffness $S_{a s m}$ (about $500 \mathrm{kN} \mathrm{m}^{-1}$ ) and results in an additional deflection $F / S_{\text {asm }}$, where $F$ is the load. The second correction is due to thermal drift. During the second loading step (section c), when the load reaches $15 \mathrm{mN}$ for the second time, a small but noticeable offset in displacement can be observed in the load-displacement curve. This offset is due to the thermal drift acting between $t_{1}$ and $t_{2}$ (Fig. 3). Applying a linear correction, we write the drift rate $r_{\text {drift }}=\left(u_{2}-u_{1}\right)$ / $\left(t_{2}-t_{1}\right)$. Finally, the displacement is expressed as

$u_{\text {tip }}=u_{\text {raw }}-u_{0}-\frac{F}{S_{\text {asm }}}-r_{\text {drift }}\left(t-t_{0}\right)$

Regarding the load, the nanoindenter is effectively loadcontrolled, which causes large loading fluctuations and ultimately catastrophic failure when the sample undergoes significant softening. For optimum repeatability, loading is carried out down to a target slope rather than up to a target load. More precisely, we monitor the slope of the loaddisplacement curve: when softening takes place, this slope decreases markedly. Loading is stopped when the loading slope falls below a preset target value. Fig. 4 illustrates load-displacement curves obtained for respectively high (a) and low (b) loading slope targets.

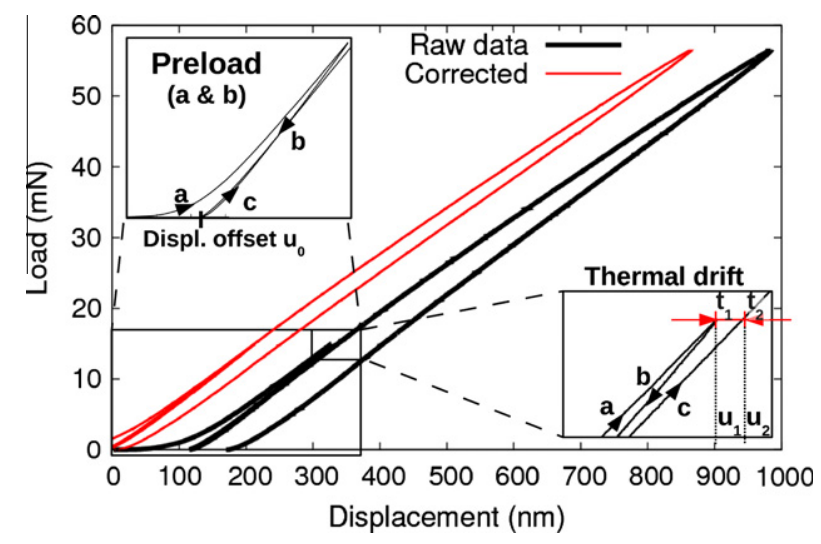

Fig. 3. Typical load-displacement curve for silica micropillar compression. A $15 \mathrm{mN}$ preload is applied to the pillar. From this preload, we correct the data for thermal drift and displacement offset. 

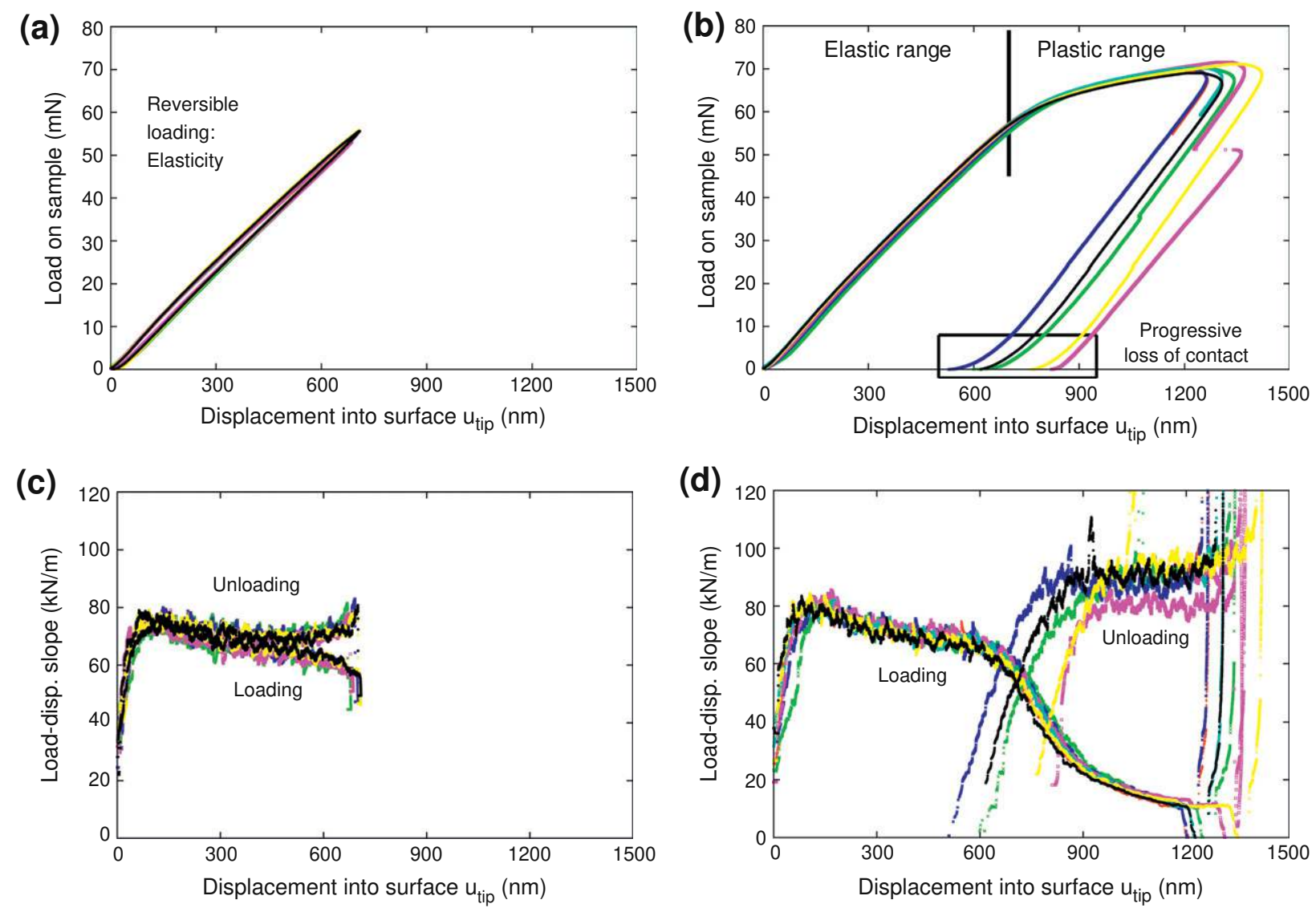

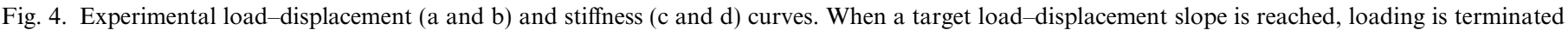
and unloading initiates. These target slopes were respectively $45 \mathrm{kN} \mathrm{m}^{-1}$ (a and c) and $10 \mathrm{kN} \mathrm{m}^{-1}(\mathrm{~b}$ and d).

\subsection{Data analysis: approximate stress-strain curves}

Before the data are compared with full FEM predictions in Section 3.2, it is useful to resort to an approximate transformation of the load-displacement curve into a stressstrain curve. An overall estimate of the axial stress and strain in the micropillar can be calculated directly assuming homogeneous uniaxial loading. This assumption would be correct if the micropillar were a perfect cylinder without friction on the upper and lower faces. The method followed here is derived from the micropillar compression literature [25]. Correcting for imperfect boundary conditions is beyond the scope of this section and will be dealt with in the FEM section. However, the taper can be taken into account approximately by expressing the axial compliance of the whole pillar $C$ (Eq. 2) as the sum of the compliances of each section

$C=\int_{0}^{h_{0}} \frac{d h}{E A(h)}=\frac{h_{0}}{E \pi r_{t o p} r_{\text {base }}}=\frac{h_{0}}{E \pi r_{e q}^{2}}$

where $r_{\text {top }}$ is the top radius and $r_{\text {base }}$ the base radius of the pillar. It appears that the radius $r_{e q}$ of the equivalent cylinder exhibiting the same stiffness as the tapered pillar can be expressed as $r_{e q}=\sqrt{r_{\text {top }} r_{\text {base }}}$.
To calculate the deformation, we note that the substrate sinks in elastically as the pillar is loaded, and this contribution to displacement must be taken into account. Following Refs. [25,29], we assume that the substrate behaves as if loaded by a rigid flat punch with a radius equal to the base radius $r_{\text {base }}$. The displacement of the pillar base $u_{\text {base }}$ follows the relation $u_{\text {base }}=F / S$ (Sneddon), where the substrate stiffness $S=2 r_{\text {base }} E /\left(1-v^{2}\right)$, and $E$ and $v$ are the Young's modulus and Poisson's ratio of the substrate. As a result, the relative displacement of the top and bottom of the micropillar is $u_{\text {eff }}=u_{\text {tip }}-u_{\text {base }}$ and the overall true strain is $\varepsilon=\ln \left(\left(h_{0}-u_{e f f}\right) / h_{0}\right)$, where $h_{0}$ is the initial height. It is worth noting that the elastic deflection of the substrate $u_{\text {base }}$ accounts for a correction of $35 \%$ of the displacement applied on the surface $u_{\text {tip }}$ in the present case.

The large elastic deformation of the pillar before yielding must also be taken into account when calculating the axial stress. Assuming a homogeneous elastic radial expansion of the equivalent cylinder, the equivalent section is $A=\pi\left(r_{e q}(1+v \varepsilon)\right)^{2}$ and the overall true stress in the equivalent cylinder is $\sigma=F / A$.

\subsection{Data analysis: finite element modeling}

An FEM model was set up to precisely identify the yield parameters best describing the experimental load- 
displacement curve, and to quantify the stress heterogeneity in the micropillar. The commercial program ABAQUS Standard 6.8 is used for that purpose [30]. An axisymmetric analysis is carried out assuming perfect alignment. The flat punch is purely elastic, described with the constants shown in Table 1. We assume a frictionless contact between the flat punch and the micropillar. The pillar itself is $4.75 \mu \mathrm{m}$ in height, with a top radius of $1.55 \mu \mathrm{m}$ and a base radius of $1.88 \mu \mathrm{m}$. It is meshed by 50 elements in height and 20 elements across the radius. The model also includes a large portion (depth $120 \mu \mathrm{m}$ and radius $60 \mu \mathrm{m}$ ) of the substrate to take the compliance into account. Analysis with fournode axisymmetric elements with reduced integration (CAX4R) was performed, accounting for the nonlinearities related to large deformations and displacements.

The constitutive model follows our previous paper [20]. The non-linear elastic response of silica [31] is not included and the elastic contribution is accounted for by a constant Young's modulus and Poisson's ratio (Table 1). We choose an elliptic yield surface in the $(p, q)$ plane (Fig. 5). This elliptic shape accounts for shear-assisted densification: densification can take place under reduced hydrostatic pressure when an additional shear load is applied [32]. The critical pressure at which densification is triggered under pure hydrostatic loading is $p_{c}$ and the critical von Mises equivalent stress generating shear flow under pure shear is $q_{c}$ (Table 1). Pressure hardening is accounted for by using the free volume (or conversely the density) as an internal variable following [20]. In this model, densification naturally saturates when the free volume vanishes.

Table 1

Parameters of the constitutive behaviors.

\begin{tabular}{lllll}
\hline Material & $\begin{array}{l}\text { Young's modulus, } \\
E(\mathrm{GPa})\end{array}$ & $\begin{array}{l}\text { Poisson's } \\
\text { ratio, } v\end{array}$ & $p_{c}(\mathrm{GPa})$ & $q_{c}(\mathrm{GPa})$ \\
\hline Amorphous silica & 70 & 0.18 & 11.5 & 7.0 \\
Diamond & 1141 & 0 & - & - \\
\hline
\end{tabular}

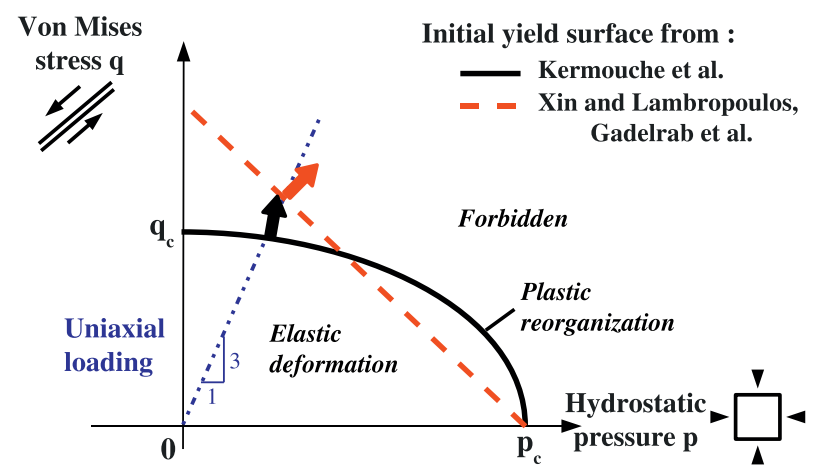

Fig. 5. Yield surface for amorphous silica. The elliptic surface used in the present paper follows Kermouche et al. [20]. Also shown is the linear criterion used by Xin and Lambropoulos [19] and by Gadelrab et al. [33]. The arrows illustrate the flow direction at first yield under uniaxial loading.
Some of the results are compared to indentation data on the same material, for which an FEM model of conical indentation of a flat surface has also been set up. The cone angle is $69.3^{\circ}$ (Vickers indenter), the indentation depth is $2 \mu \mathrm{m}$ (load $400 \mathrm{mN}$ ) and an axisymmetrical domain of depth $120 \mu \mathrm{m}$ and radius $60 \mu \mathrm{m}$ is considered.

\section{Results}

\subsection{Experimental results}

The raw data for the compression of silica pillars are shown in Fig. 4a for high target slope. Under this condition, the maximum load is slightly below $60 \mathrm{mN}$ and full elastic recovery of the pillar is found after unloading. The slope of the load-displacement curve during unloading is very close to the slope during loading (Fig. 4c).

For a low target slope, softening is observed around $F=60 \mathrm{mN}$ and a permanent height reduction is observed in the load range $60-70 \mathrm{mN}$ (Fig. 4b). At $70 \mathrm{mN}$ load, the permanent height reduction is about $800 \mathrm{~nm}$. Simultaneously, we observe that the slope of the load-displacement curve at unloading has increased noticeably (Fig. 4d).

Note that the preload has successfully removed any progressive development of the contact (Fig. 4a and b) upon loading, demonstrating that minimal misalignment between punch and pillar is achieved with the tilt stage [29]. More generally, in both cases, a very satisfactory reproducibility of the measurements is achieved thanks to the protocol outlined in Section 2.

The raw data have also been transformed into stressstrain curves, as detailed in Section 2. For a high target slope the maximum axial stress is $6.5 \mathrm{GPa}$, while for low target slopes irreversible deformation occurs in the range 6.5-8 GPa (Fig. 6a). At maximum load, with a residual deformation of $800 \mathrm{~nm}$, the true strain reaches 0.2 , which is an $18 \%$ engineering strain.

A scanning electron microscopy (SEM) image of a pillar after irreversible deformation is shown in Fig. 6b. Interferometry measurements (ZYGO NewView 5032) confirm that the height of the pillars has been reduced by $800 \mathrm{~nm}$. From the SEM picture, it clearly appears that the upper section of the pillar has undergone a significant permanent deformation which involves radial expansion. In addition regularly spaced radial cracks can be seen at the periphery of this zone, either significantly developed (Fig. 6b), or just initiated (Fig. 6c). We also observe that after permanent deformation the top surface is often tilted by a few degrees with respect to the vertical axis.

Finally, catastrophic failures are sometimes observed during unloading, as visible on two of the seven data sets displayed in Fig. 6a. SEM observations reveal that in this case two failure morphologies may be identified. When failure is partial, the pillar is split across by a vertical fracture (Fig. 6d). For full rupture, the pillar is wiped out of the surface (not shown), probably due to failure at the pillar base, along the horizontal plane. 

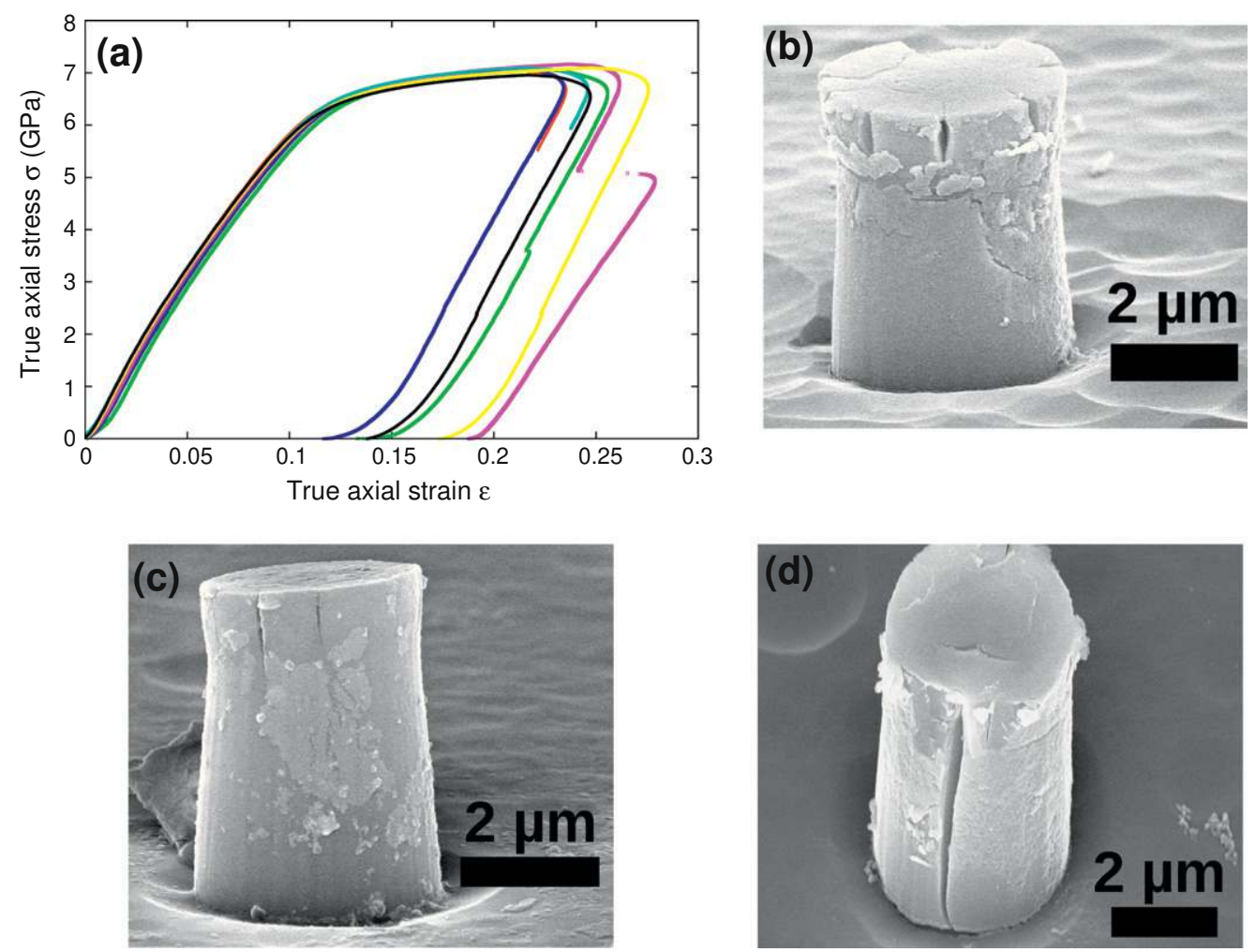

Fig. 6. (a) True stress and true strain plots for seven individual pillars derived from Fig. 4b. (b), (c) and (d) are FEG-SEM observations of individual micropillars after irreversible deformation.

\subsection{Numerical results}

A typical load-displacement curve measured in uniaxial compression with a low slope target (high load) is compared to the FEM prediction in Fig. 7a. Below $60 \mathrm{mN}$ a reversible softening is observed experimentally (Fig. 4c) because of the non-linear elastic response of silica. This behavior is not reproduced by the model because the elastic non-linearity was omitted. However, permanent flow initiates at a load close to $60 \mathrm{mN}$ both in the experiments and in the model, and develops up to ca. $70 \mathrm{mN}$ over an irreversible displacement, which is slightly smaller $(600 \mathrm{~nm})$ in the FEM calculation than in the measurements $(800 \mathrm{~nm})$.

The von Mises equivalent stress field predicted by the FEM calculations for a $65 \mathrm{mN}$ load, just above yield, is displayed in Fig. $7 \mathrm{~b}$. When the yield initiates, the von Mises equivalent stress reaches $6.85 \mathrm{GPa}$ at the top of the pillar. The distribution of $(p, q)$ stress states in the micropillar is displayed in Fig. 7c and d, along with the initial yield surface. For a uniaxial loading we expect $q / p=3$, and this straight line is shown for comparison.

To understand cracking, the distributions of circumferential stress (or hoop stress $\sigma_{\theta \theta}$ ) are displayed in Fig. 8 for three load levels: at the beginning of the plastic flow (a), the maximum load (b) and after unloading (c).

For comparison, we also calculated stress distributions for cone indentation at a $400 \mathrm{mN}$ load with the same con- stitutive behavior. A comparison between the experimental and predicted load-displacement curves is shown in Fig. 9a. The von Mises equivalent stress field is shown in Fig. 9b. The stress state distribution for a $400 \mathrm{mN}$ load is shown in Fig. 9c, and the stress state history for four elements located at different depths under the indenter tip is shown in Fig. 9d. Finally, the distribution of the circumferential stress is displayed in Fig. 10 under load (left) and after unloading (right).

\section{Discussion}

\subsection{Elastic response: plastic flow}

The permanent height reduction observed above $60 \mathrm{mN}$ on the loading-unloading curves (Fig. 4b) along with the permanent increase in the axial stiffness (Fig. 4d at unloading) demonstrate that above the $60 \mathrm{mN}$ threshold the deformation of the micropillar is plastic in nature. Indeed, significant damage, which could also result in irreversible deformation, would reduce the stiffness in the loading direction instead. The SEM observations clearly indicate that the initially tapered upper section of the pillars flares up after plastic deformation (Fig. 6b-d), which is evidence of significant plastic shear flow. It is also worth noting that no visible slip band is found on the plastically deformed pillars by SEM inspection. This plastic flow often leads to an asymmetric deformation of the pillar (Fig. 6c), 
(a)

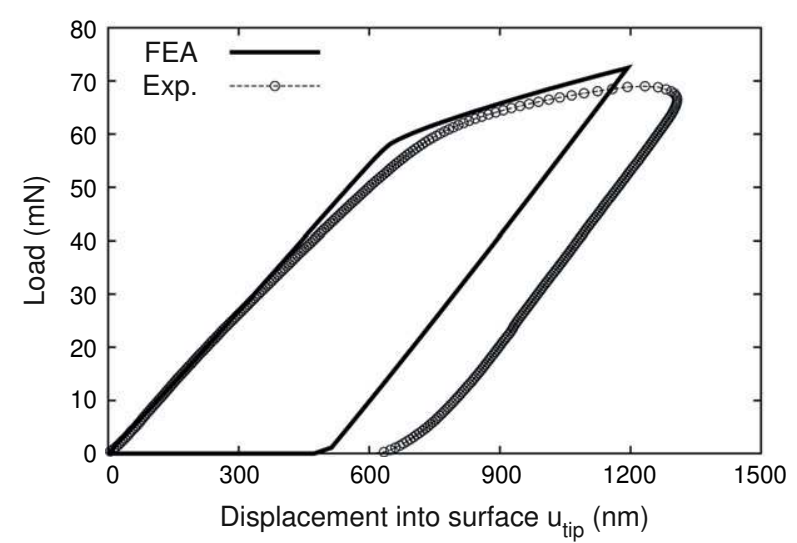

(c)

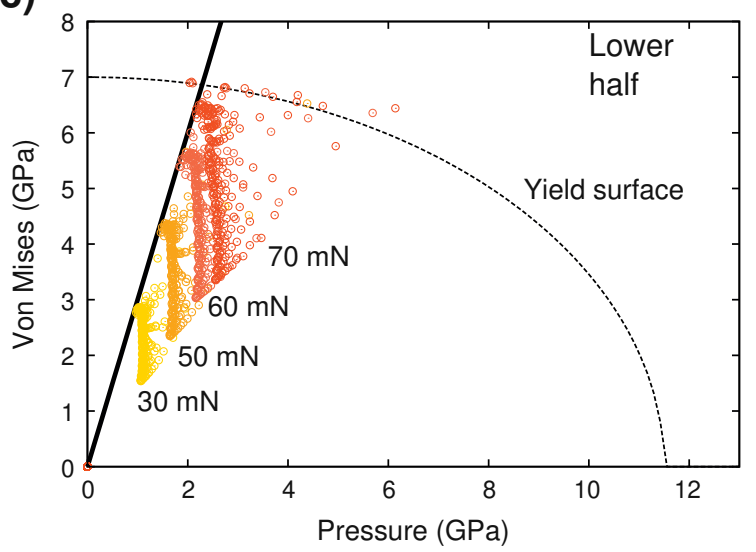

(b)

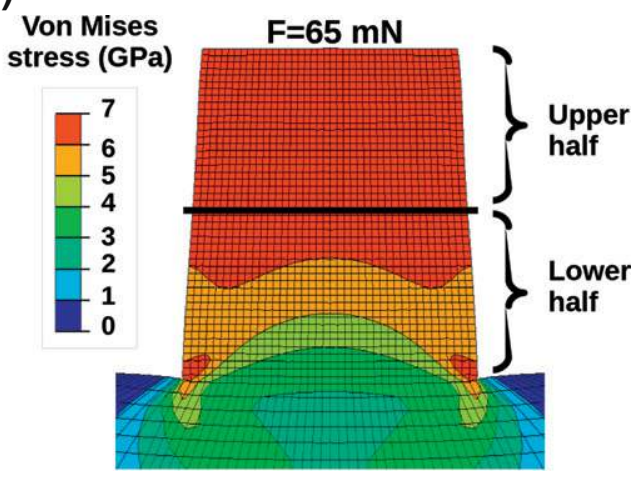

(d)

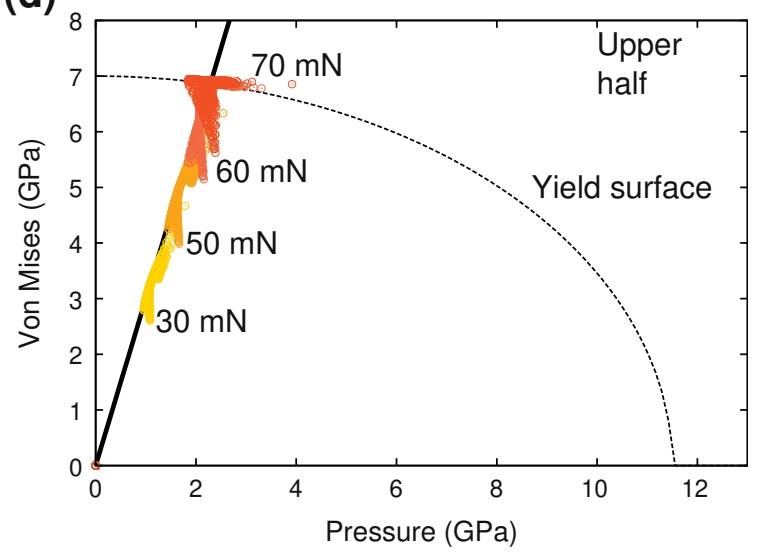

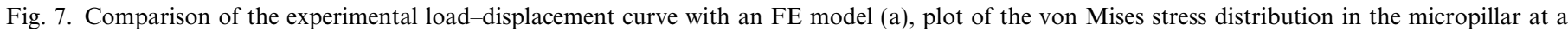

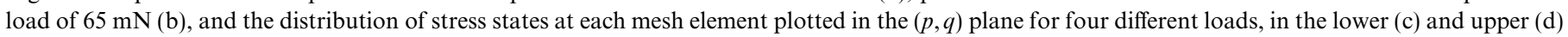
half of the micropillar. The solid line shows the ideal uniaxial loading.

resulting in a slanted top surface after deformation, as evidenced both by SEM and interferometric measurements. The resulting misalignment of the top surface is consistent with the strong curvature of the stress-strain curve in the final stage of unloading, over a range of about $200 \mathrm{~nm}$ (Fig. 4b), which reflects the progressive loss of contact between punch and pillar.

\subsection{Pillar geometry and deviation from ideality}

The FEM simulations can help assess the extent to which a uniaxial stress state $(q / p=3)$ holds true in the micropillar, during both the elastic and plastic regimes. The micropillar is perfectly bonded to the substrate, and this constrains the radial displacements of the base. The resulting confinement leads to higher levels of hydrostatic pressure in that region, as evidenced by our FEM simulations (Fig. 7c and d). This slight enhancement of the hydrostatic pressure is the main deviation from ideal uniaxial loading. In the upper half of the pillar the constraint from the substrate is minimal and $q / p \simeq 3$, as expected for uniaxial loading (Fig. 7d).

Because the pillar tapers, the section is larger in the lower part of the pillar, reducing $q$ at the base: the yield threshold is first reached in the upper part of the pillar, where the plastic deformation localizes. The variation in the section over the height of the pillar also results in a slight discrepancy between our estimate (Section 2.3) of the average axial stress $(6.5 \mathrm{GPa})$ and the actual yield stress (6.85 $\mathrm{GPa}$ in the upper region) at the threshold inferred from the FEM calculations.

The SEM observations show that the top surface of the pillar exhibits the largest radial deformation (Fig. 6b). Significant friction between the punch and the pillar would constrain the radial displacement of this surface [28]; however, minimal friction is thought to take place, thus qualitatively validating our assumption of frictionless contact for the FEM calculations.

\subsection{Quantification of the constitutive equation}

During loading, the yield criterion is first reached at elements located in the upper region of the micropillar, where $q / p \simeq 3$. It is remarkable that the same relation still holds quite accurately during plastic flow at loads well above threshold (Fig. 7c and d). This observation is especially true in the upper region of the pillar, where substrate confinement is absent and minimal hydrostatic compression 

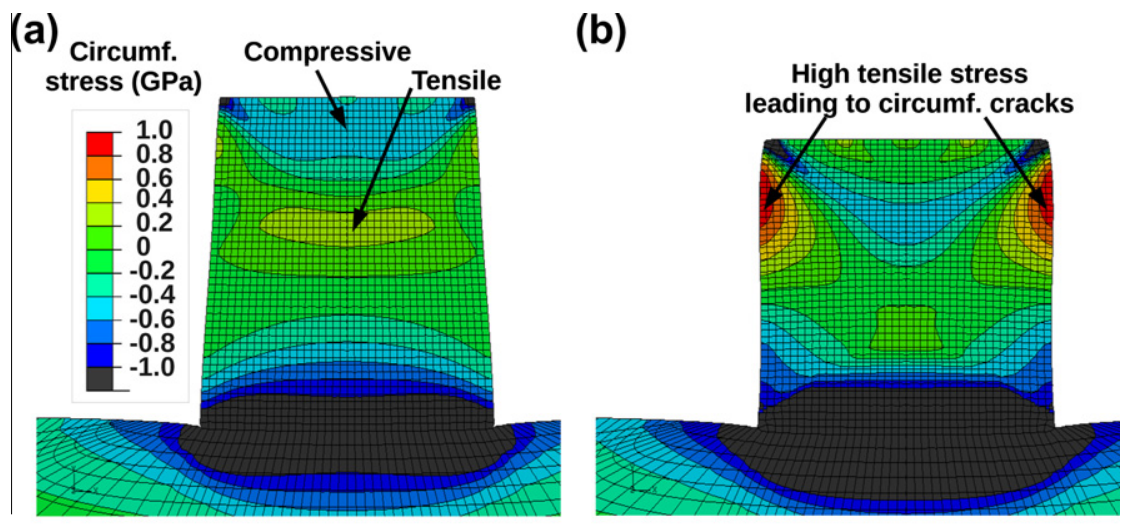

(c) High residual tensile stress

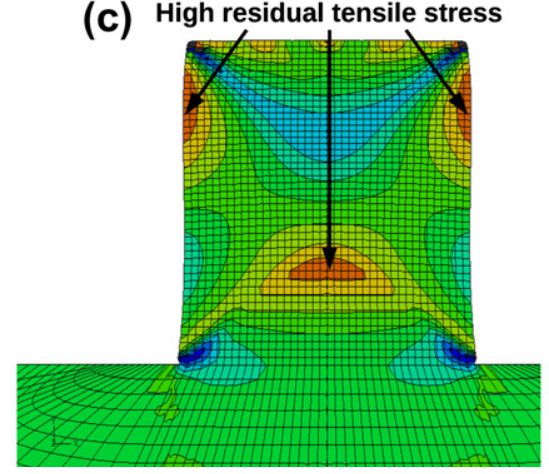

Fig. 8. FEM calculations of the circumferential stress in the micropillar at $60 \mathrm{mN}$ load (a), $75 \mathrm{mN}$ load (b) and after unloading (c).

develops during pillar compression, even in the plastic regime.

The strain hardening included in the present model of plastic flow (Section 2.4) is controlled by the volumetric plastic deformation or densification. For micropillar compression, our calculations predict that densification does not exceed $4 \%$, and plastic deformation is reasonably close to the shear flow expected for a perfectly plastic material under ideal uniaxial loading, especially in the upper half of the pillar. As a result, in the compression of tapered micropillars, plastic flow has a stabilizing effect. From the expansion of the carrying section there appears an apparent hardening: the pillar can bear loads larger than threshold while the material itself experiences hardly any strain hardening (Fig. 7d).

Based on our FEM calculations, we conclude that the overall axial stress in the micropillar (6.5 GPa at threshold) is best accounted for by taking a yield stress in shear $q_{c}=7 \mathrm{GPa}$ (Table 1). This value is only slightly larger than the $6.5 \mathrm{GPa}$ initially determined in Ref. [20]. With this value, the yield point in pure uniaxial compression is $6.85 \mathrm{GPa}$ (Fig. 5), which is in between the values reported in the literature (6.4 GPa [20] and 8.3 GPa [33]).

It is notable that this value is within the range of the intrinsic tensile strengths measured on pristine silica fibers and actually lies in between the values for accepted room temperature and the values for inert conditions $[4,5]$. Since both uniaxial compression and uniaxial tension are dominated by equivalent von Mises stress, a similar yield stress could indeed be expected in these two kinds of loadings. Moreover, if similar plastic events are involved in compression and tension, opposite macroscopic behaviors are expected: stabilization by expansion of the section for compression and destabilization by necking for traction. Validating such a conjecture would require further investigations into the tensile strength of silica fibers.

\subsection{Comparison with indentation}

For comparison, we also calculated the stress and strain distributions for cone indentation using the same constitutive model. Good agreement with the data is obtained, as demonstrated by the comparison between the experimental and predicted load-displacement curves (Fig. 9a). Cone indentation is a self-similar problem, and the stress maps for one prescribed depth (Fig. 9b) account for the full loading curve. The stress state distribution for a $2 \mu \mathrm{m}$ depth has been plotted in Fig. 9c. In this distribution, two distinct sets of local stress states are evident. We observe that the $q / p$ ratio for all elements in the elastic regime falls around 4, which is quite similar to the pillar distribution, especially in the upper part of the pillar, where the slope is 3 . For elements in the plastic regime, in cone indentation, $q$ stays close to $q_{c}$ and a large range of hydrostatic pressures can be explored (Fig. 9c). This wider range of hydrostatic pressures in the plastic regions is the major contrast with the pillar (Fig. 7c), where the stress state remains nearly uniaxial even in the plastic regime. 

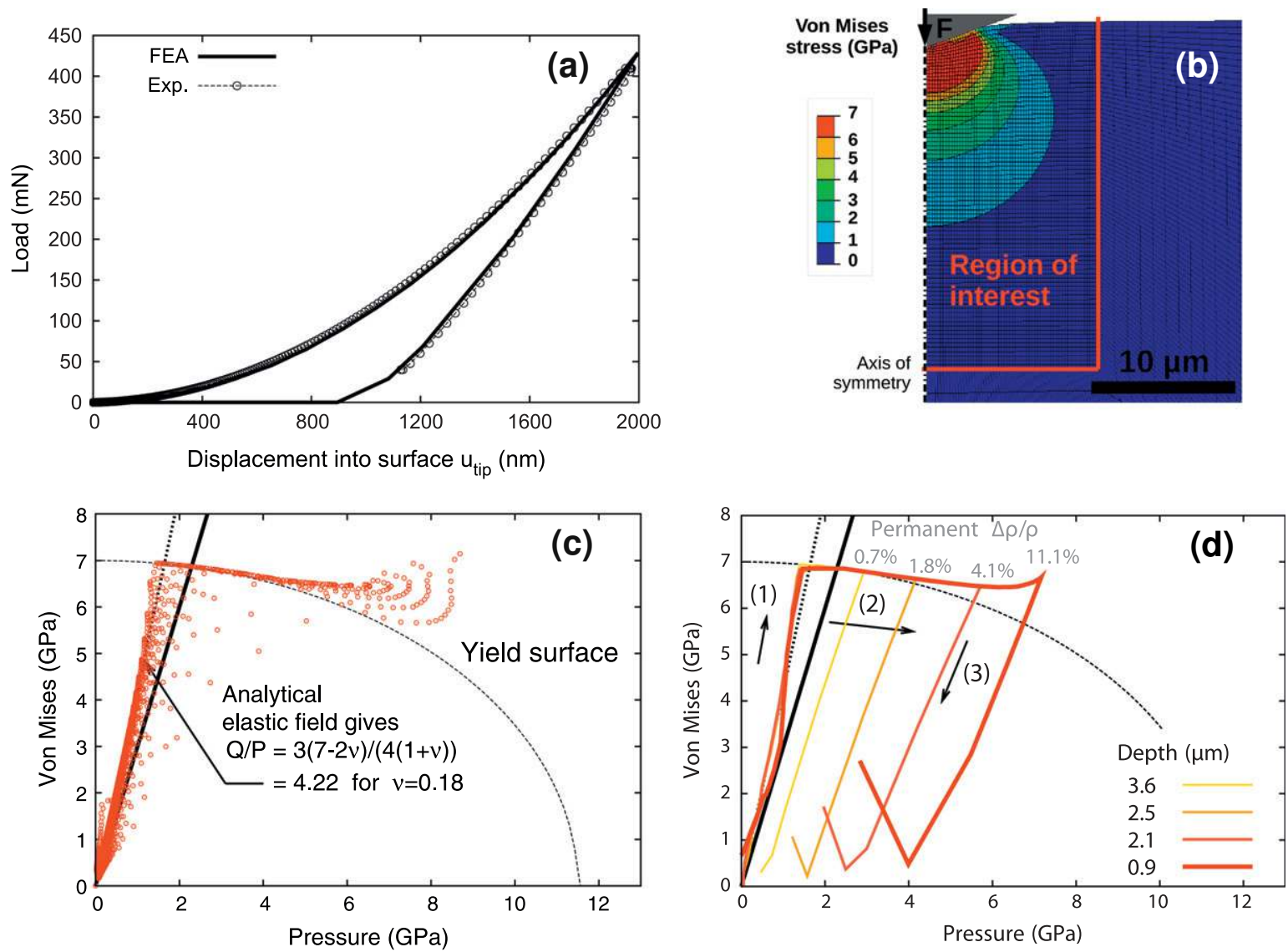

Fig. 9. Cone indentation in silica: experimental and FEM load-displacement curves (a), von Mises stress at a $400 \mathrm{mN}$ load (b), distribution of stress states at each mesh element of the region of interest plotted in the $(p, q)$ plane (c), stress state trajectories for four elements located at different depths under the indenter tip during the full loading-unloading cycle.

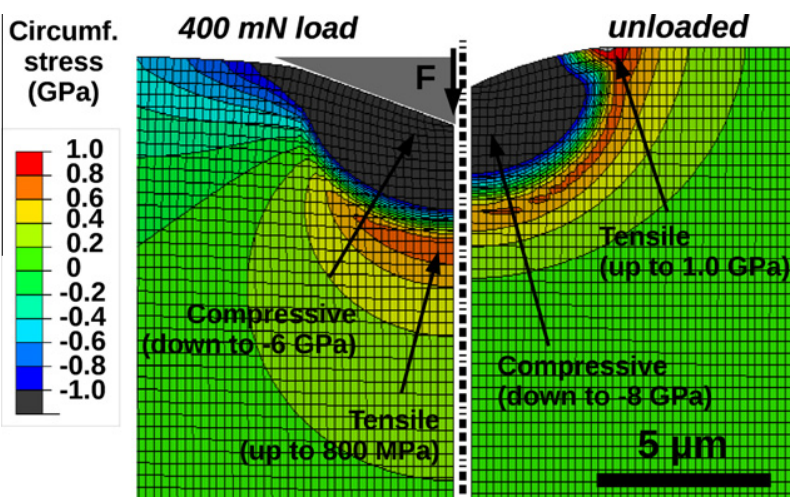

Fig. 10. FEM calculations of the circumferential stress in the median plane of a conical indent in silica at a $400 \mathrm{mN}$ load (left), and after unloading (right).

It is also instructive to track the local stress state of an individual element during a full cone indentation cycle. The stress trajectories for points located at various depths under the indenter tip are illustrated in Fig. 9d. In the elastic regime the load rises with a constant ratio $q / p$ around 4 (Fig. 9d, segment 1). Once the yield surface has been reached, shear flow occurs, but a gradual increase in the hydrostatic pressures also develops, as demonstrated by the nearly horizontal portion of segment 2 . It is worth noting that this increase in hydrostatic pressure results in the significant densification that has been shown experimentally for indentation. In line with the requirement of selfsimilarity, we consistently observe that the hydrostatic pressure is larger for elements closer to the indenter tip, and so is the permanent densification $\Delta \rho / \rho$ (Fig. 9d, in gray). Finally, during unloading (segment 3 ), $q$ first decreases but can rise again at low loads as residual stresses build-up due to permanent strain.

For a better understanding of the elastic loading phase in cone indentation, we investigate the $q / p$ ratio in the far field of an indent. Far enough from the indenter, and ignoring the local effects of the indenter geometry, we can use the analytical expressions for the elastic stress field resulting from normal point loading at the surface [34]. When considering a region located under the indenter along the loading axis, we find

$q / p=\frac{3(7-2 v)}{4(1+v)}$

We also find that the same relation still holds quite accurately when moving away from the $z$-axis. In the case of amorphous silica, Eq. 3 predicts that the $q / p$ ratio under the indenter in the elastic regime is 4.22 for cone 
indentation, which is in good agreement with the $q / p$ ratio evidenced from the FE model (Fig. 9c and d, dashed line). Note also that for an incompressible material a uniaxial stress state appears under normal point loading $(q / p=3$ for $v=0.5$ ).

In summary, we find that the yield surface is first reached at very similar values of hydrostatic pressure and von Mises stress both in pillar compression and in cone indentation. In particular, cone indentation does not lead to higher levels of hydrostatic pressure in the elastic regime. A strong contrast between pillar compression and cone indentation appears only in the plastic regime. Indeed, in cone indentation, plastic shear flow is confined by the surrounding elastic medium, resulting in the build-up of hydrostatic pressure shown in Fig. 9d, segment 2. By cutting out material around the pillar, we suppress this confinement and plastic shear flow can develop while preserving the uniaxial stress state. Altogether, our interpretation of densification during cone indentation of silica is as follows: volume-conservative flow is the main driving force for densification through the build-up of hydrostatic pressure resulting from confinement.

\subsection{Flow direction}

In this comparison with cone indentation, our interpretation strongly depends on the choice of the flow direction, which is the ratio of densification to shear flow, as marked by arrows in Fig. 5. Here we have simply assumed associated plasticity, and with the elliptic form of the yield surface, this flow direction results in nearly pure shear flow under uniaxial stress. A flow including more intrinsic densification would result in a moderate build-up of hydrostatic pressure. However, numerical investigations of various intrinsic flow directions could all lead to similar permanent densifications under sharp indentation, either from the intrinsic flow direction or by constrained shear flow. Densification measurements by Raman spectroscopy on sharp indentation imprints do not seem appropriate for assessing the actual direction of intrinsic flow at first yield. In that aspect, micropillar compression appears to be a more interesting alternative, as the potentially observed densification would mainly be related to the intrinsic flow direction. More precise investigations based on Raman measurements of the densification at the top of the pillar as well as comparisons of the profile of deformed micropillars with FEM models with different yield directions could provide some insight into this question.

\subsection{Residual stress field}

The inhomogeneous permanent deformation due to plastic flow induces residual stresses. It is well known that stress fields in brittle materials like silicates transpire in the fracture patterns [35]. Here we discuss the nature of the stress fields in pillars under compression as well as the residual stress fields after unloading. We also sketch a brief comparison with stress distributions in cone indentation.

In pillar compression below the threshold, the circumferential stresses are compressive. They are small but finite (not shown) because the base of the pillar is constrained by the substrate. When the load reaches the threshold, plastic flow is triggered in the upper region, starting from the upper edge. Material flows along the directions of maximal equivalent von Mises stress, diagonally across the pillar. This flow results in a radial expansion of the upper part of the pillar. For loads slightly above the yield threshold, the expansion of this nucleus leads to the build-up of significant compressive circumferential stresses (Fig. 8a) in the upper part of the pillar (about $-0.5 \mathrm{GPa}$ ) and also strains the lower, elastic, part of the pillar, which becomes tensile (about $+0.5 \mathrm{GPa}$ ). At higher loads the plastic deformation wedges into the upper part of the pillar. The compressive circumferential stresses increase further, reaching $-1 \mathrm{GPa}$ at the upper corners (Fig. 8b). The tensile restraining stresses also build up, but their location shifts towards the edges: in particular, our calculations predict a restraining annulus of tensile stresses at the periphery, with a maximum value $+1 \mathrm{GPa}$ located at about $1 \mu \mathrm{m}$ under the upper edge of the pillar. Furthermore, the locations of tensile stress presented here are consistent with the FE analysis of Schwaiger and co-authors on the deviation from the purely uniaxial stress state during micropillar compression [28].

We can conclude from this analysis, together with the observations of radial cracks at this exact location (Fig. 6b and c), that the circumferential tensile stress produces a decohesion of the strained silica. This decohesion appears to initiate at stresses under $1 \mathrm{GPa}$, at the beginning of the plastic flow resulting from the axial stress (Fig. 6c). This level of stress is significantly lower than the tensile strength obtained in fibers, for instance. Indeed, tensile stresses of 5-6 GPa can be reached before failure of pristine fibers at room temperature in ambient air $[4,5]$. The decohesion observed in the present experiments could result from a weakening of the amorphous network induced by plastic reorganization, as has been proposed on the basis of molecular dynamics simulations [36].

When the load is removed after plastic flow has taken place in the upper part of the pillar, there is a significant residual radial deformation. However, the base is now relieved of radial compressive stresses and a median region of tensile stresses appears halfway up the pillar, just below the plastically deformed region (Fig. 8c). We think these residual stresses may be responsible for the catastrophic failure sometimes observed during unloading. A split pillar (Fig. 6e) may result from a crack originating from the tensile circumferential stresses in the median region, as Howie and co-authors have previously observed in $\mathrm{Si}$ and $\mathrm{InAs}$ micropillars [24].

There is some similarity between the stress fields described here for a pillar and the stress fields for cone indentation. For cone indentation under load (Fig. 10, 
left), the elastic and plastic contributions result in a large tensile circumferential stress in the median region below the plastically deformed core [35]. However, there is also a restraining effect due to confinement by the surrounding medium, and the circumferential stress at the surface is compressive. Indeed, in normal glasses, median cracks, located under the plastically deformed area, initiate during loading. This result can be contrasted with our observations on pillars: the upper part of the pillar is not confined, but the base of the pillar is. As a result, the tensile region shifts to the upper region of the pillar sides. This is the region where we observe the array of radial cracks.

In cone indentation, after unloading, the compressive contribution from the elastic field is zero and the tensile circumferential stress distribution at the surface due to the plastic flow of the core region becomes apparent (Fig. 10.right). Indeed, in normal glasses, which experience a quite similar stress distribution, radial cracks initiate upon unloading at the surface from the edge of the plastically deformed core [35]. For pillars, the confinement effect is limited to the base of the pillar and the elastic field does not induce tensile circumferential stresses there. As a result, the tensile area under the plastically deformed core appears only upon unloading (Fig. 8c), as does the tensile region at the base of the pillar, inducing the catastrophic rupture already mentioned.

In brief, in pillars, substrate confinement effects are much more limited and only affect the base of the pillar, while in cone indentation, substrate confinement is more pronounced at the surface. Due to this contrast, the respective positions of tensile and compressive circumferential stresses under load are inverted: while median cracks appear in cone indentation, we observe an array of radial cracks at the top of the pillar. After unloading, the stress distribution becomes essentially tensile all around the plastically deformed core in both cases: radial cracks develop in cone indentation, while we ascribe a split pillar morphology to cracks originating from this distribution.

\section{Conclusions}

We have demonstrated stable plastic flow in micronsized silica pillars. Due to the slightly tapered shape, irreversible deformation is dominated by radial flow in the upper part of the pillar, resulting in a flare at the top of the pillar. The plastic deformation reaches $20 \%$. This is accompanied by a well-defined crack pattern: a periodic array of radial cracks form at the top edge of the pillar. A few of the pillars also split into two upon unloading.

We have shown that these results are fully consistent with the constitutive equation we proposed earlier [20]. We propose here a single minor adjustment by raising $q_{c}$ from 6.5 to $7 \mathrm{GPa}$. This value is in the range of the intrinsic tensile strength values measured on pristine silica fibers. The intrinsic tensile strength could therefore involve some plasticity, although no definite evidence can yet be brought in that regard.
Deviations from ideal uniaxial loading have also been carefully analyzed. We contend that, for silica, an adequate geometry is a pillar with a 1.5 aspect ratio: in this case, an almost purely uniaxial loading is effectively obtained in the upper half of the pillar. A significantly longer pillar would buckle away during compression; a significantly shorter one would be dominated by substrate effects. Our analysis also places bounds on the errors incurred through an approximate analytical analysis of the raw data.

Under the present assumptions (elliptic yield surface and associated plasticity), we have characterized the plastic deformation mechanisms. We conclude that, in cone indentation, volume-conservative shear flow indirectly induces densification: as the flow is constrained by the strong confinement, significant hydrostatic pressure builds up, resulting in densification. In contrast, in the uniaxial compression of micropillars, plastic flow is free, with reduced hydrostatic pressure and minimal densification. These considerations open the way for a more accurate quantification of the flow direction through: (i) precise shape analysis of the micropillar; and (ii) direct measurement of densification through Raman spectroscopy.

Along the same lines, we analyzed confinement as the origin of the contrasted stress distributions calculated for pillar compression and for cone indentation, both under load and after unloading. We found the results to be in good agreement with the observed crack pattern: in particular, the radial cracks at the top of the pillar are consistent with the annulus of restraining circumferential stresses we found at this location by FEM calculations. The magnitude of these tensile stresses (below $1 \mathrm{GPa}$ ) suggests that decohesion occurs under stresses considerably lower than expected when considering the tensile strengths of pristine fibers. We surmise that this discrepancy is due to the strong plastic deformation of the material. This weakening of the amorphous network during plastic flow might be an interesting starting point for studying damage initiation in silica.

\section{Acknowledgements}

The authors thank the FEMTO-ST Institute for manufacturing the micropillars within the MIMENTO technologic platform, as well as Gaylord Guillonneau (LTDS) and Didier Pellerin (Scientec) for fruitful interactions.

\section{References}

[1] Taylor EW. Nature 1949;163(4139):323.

[2] Marsh DM. Proc R Soc London A 1964;279(1378):420-35.

[3] Lawn BR. Fracture of brittle solids. Cambridge University Press; 1993.

[4] Proctor BA, Whitney I, Johnson JW. Proc R Soc A 1967;297:534-57.

[5] Kurkjian CR, Gupta PK, Brow RK, Lower N. J Non-Cryst Solids 2003;316(1):114-24.

[6] Rodney D, Tanguy A, Vandembroucq D. Model Simul Mater Sci Eng 2011;19:083001.

[7] Argon AS. Acta Metall Mater 1979;27(1):47-58.

[8] Tsamados M, Tanguy A, Goldenberg C, Barrat JL. Phys Rev E 2009;80(2):026112. 
[9] Ernsberger FM. J Am Ceram Soc 1968;51(10):545-7.

[10] Seifert FA, Mysen BO, Virgo D. Phys Chem Glasses 1983;24(6):141-5.

[11] Susman S, Volin KJ, Liebermann RC, Gwanmesia GD, Wang YB. Phys Chem Glasses 1990;31(4):144-50.

[12] Hagan JT. J Mater Sci 1980;15(6):1417-24.

[13] Polian A, Grimsditch M. Phys Rev B 1990;41(9):6086-7.

[14] Perriot A, Vandembroucq D, Barthel E, Martinez V, Grosvalet L, Martinet C, et al. J Am Ceram Soc 2006;89(2):596-601.

[15] Vandembroucq D, Deschamps T, Coussa C, Perriot A, Barthel E, Champagnon B, et al. J Phys: Condens Matter 2008;20(48):485221.

[16] Ji H, Keryvin V, Rouxel T, Hammouda H. Scripta Mater 2006;55:11591162.

[17] Deschamps T, Martinet C, Bruneel JL, Champagnon B. J Phys: Condens Matter 2011;23:035402.

[18] Rouxel T, Ji H, Guin JP, Augereau F, Rufflé B. J Appl Phys 2010;107(9):094903.

[19] Xin K, Lambropoulos JC. Proc SPIE 2000;4102(1):112.

[20] Kermouche G, Barthel E, Vandembroucq D, Dubujet P. Acta Mater 2008;56(13):3222-8.

[21] Uchic MD, Dimiduk DM. Mater Sci Eng: A 2005;400-401(0):268-78.

[22] Volkert CA, Donohue A, Spaepen F. J Appl Phys 2008;103(8).
[23] Maaß R, Uchic MD. Acta Mater 2012;60(3):1027-37.

[24] Howie PR, Korte S, Clegg WJ. J Mater Res 2012;27(1):141-51.

[25] Zhang H, Schuster BE, Wei Q, Ramesh KT. Scripta Mater 2006;54(2):181-6.

[26] Yang Y, Ye JC, Lu J, Liu FX, Liaw PK. Acta Mater 2009;57(5):1613-23.

[27] Bharathula A, Lee SW, Wright WJ, Flores KM. Acta Mater 2010;58(17):5789-96.

[28] Schwaiger R, Weber M, Moser B, Gumbsch P, Kraft O. J Mater Res 2012;27(1):266-77.

[29] Lacroix R, Chomienne V, Kermouche G, Teisseire J, Barthel E, Queste S. Int J App Glass Sci 2012;3(1):36-43.

[30] Abaqus Analysis User's manual, Version 6.8. Dassault Systemes Simulia Corp.; 2010.

[31] Kondo KI, Iio S, Sawaoka A. J Appl Phys 1981;52(4):2826-31.

[32] Mackenzie JD. J Am Ceram Soc 1963;46(10):461-70.

[33] Gadelrab KR, Bonilla FA, Chiesa M. J Non-Cryst Solids 2012;358(2):392-8.

[34] Yoffe EH. Phil Mag A 1982;46(4):617-28.

[35] Lawn BR, Evans AG, Marshall DB. J Am Ceram Soc 1980;63(9-10):574-81.

[36] Taniguchi T, Ito S. J Ceram Soc Jpn 2008;116(1356):885-9. 